\title{
Etnobotânica e medicina popular no tratamento de malária e males associados na comunidade ribeirinha Julião - baixo Rio Negro (Amazônia Central)
}

VEIGA, J.B.*; SCUDELLER, V.V. ${ }^{2}$

1 Instituto Nacional de Pesquisas da Amazônia (INPA), Coordenação de Dinâmica Ambiental Av. André Araújo, 2936, Petrópolis, CEP: 69080-971, Manaus-AM; 2 Universidade Federal do Amazonas, ICB/ Depto Biologia (Av. General Rodrigo Octávio, 6200, Coroado I, CEP: 69077-000, Manaus-AM), scudellerveridiana@hotmail. com. *Autor para correspondência: josephinaveiga@gmail.com

RESUMO: A utilização de plantas medicinais para o tratamento de doenças tropicais como a malária na Amazônia Central é de suma importância, principalmente em locais onde o sistema único de saúde não se encontra presente como na maioria das comunidades ribeirinhas desta região. Sendo assim, investigar e resgatar o conhecimento popular a respeito de plantas medicinais utilizadas no tratamento de malária e males associados pelos moradores da comunidade Julião situada na Reserva de Desenvolvimento Sustentável do Tupé, Manaus-AM, torna-se importante no registro de como as populações locais se previnem e tratam essa doença tão prevalente e perigosa na região. O trabalho foi conduzido na forma de oficinas participativas, segregadas por gênero e complementadas com entrevistas semiestruturadas aliadas à técnica da turnê-guiada nos quintais e floresta adjacente à comunidade. Foram calculados os índices de diversidade de Shannon-Wiener, equitabilidade e concordância quanto ao uso principal (CUP). A partir da colaboração efetiva de 13 comunitários foram registradas 62 espécies vegetais pertencentes a 53 gêneros e 34 famílias botânicas que resultaram em índice de diversidade $\left(H^{\prime}\right)$ de 1,62 decits e equitabilidade de 0,9. As famílias mais representativas foram: Fabaceae (7 espécies), Asteraceae e Lamiaceae (4 espécies cada) e Solanaceae e Rutaceae (3 espécies cada). Vale destacar que 16 espécies $(25,8 \%)$ foram citadas para tratamento de malária e males associados pela primeira vez em estudos etnobotânicos realizados na América Latina.

Palavras-chave: conhecimento tradicional, plantas medicinais, Reserva de Desenvolvimento Sustentável do Tupé, ribeirinhos, doenças negligenciadas

\begin{abstract}
Ethnobotany and popular medicine in the treatment of malaria and associated diseases in theriverside community in Julião-Low Black River (Central Amazonia). The use of medicinal plants in order to treat tropical diseases such as malaria is of extreme importance, particularly in places where the public health system is not present as in most coastal communities of the Amazonia region. That being stated, investigating and rediscovering the popular knowledge of medicinal plants used to treat malaria and its associated diseases by the residents of the Julião community, located in RDS Tupé, Manaus-AM, becomes relevant in recording how local populations prevent and treat this disease so prevalent and dangerous in this region. In order to carry out this study, it was implemented an ethnobotanical survey through workshops organized in categories and semi-structured interviews as well as guided tours by residents of the community in their backyards and nearby forest areas. The ShannonWiener diversity indicators were calculated, measuring equitability and concurrence regarding its main use (CUP). From the effective collaboration of 13 community members, 62 plant species belonging to 53 types and 34 botanic families were observed, which resulted in a diversity index $\left(\mathrm{H}^{\prime}\right)$ and equitability of 1.62 decits 0.9 . The most significant botanical families shown in the survey were: Fabaceae (7 species), Asteraceae (4 species), Lamiaceae (4 species), Solanaceae ( 3 species) and Rubiaceae ( 3 species). It is worth to highlight that 16 species $(25.8 \%)$ were mentioned for malaria treatment and associated diseases, for the first time in ethnobotanical studies conducted in Latin America.
\end{abstract}

Keywords: traditional knowledge, medicinal plants, Sustainable Development Reserve Tupé, riparian, neglected diseases 


\section{INTRODUÇÃO}

Embora a relação entre populações humanas e recursos vegetais tenha sido abordada desde a antiguidade, o termo etnobotânica foi cunhado há pouco mais de um século, originalmente como o estudo das plantas usadas por povos primitivos e aborígenes por Harshberger (Plotkin, 1995; Balick \& Cox, 1997). Neste contexto, estudos etnobotânicos são importantes principalmente no Brasil, uma vez que o seu território abriga uma das floras mais ricas do globo, da qual $99,6 \%$ são desconhecidas quimicamente (Gottlieb et al., 1996) e também uma das maiores diversidades culturais (Revilla, 2002).

A prática da medicina tradicional está inclusa e reconhecida no sistema primário de saúde em países em desenvolvimento (Cunningham, 1993; Balick et al., 2000). A Organização Mundial de Saúde (OMS) estima que cerca de $80 \%$ da população mundial depende de plantas para o cuidado com a saúde e relata ainda que $85 \%$ da medicina tradicional envolvem o uso de plantas, seus extratos vegetais e seus princípios ativos. Isso significa que 3,5 a 4,0 bilhões de pessoas dependem de plantas como fontes de drogas. No Brasil, as plantas medicinais foram oficialmente incluídas no SUS (Sistema Único de Saúde), a partir de um conjunto de resoluções e portarias que delineiam os instrumentos necessários à implantação da Política Nacional de Plantas Medicinais e Fitoterápicos destacando-se a Relação Nacional de Plantas Medicinais de Interesse ao SUS (Brasil, 2009). No entanto, poucas cidades realmente aderiram ao programa e a maioria das plantas utilizadas é exótica.

As plantas medicinais na região amazônica representam a principal forma de tratamento de doenças para a maioria das populações pobres devido às influências culturais e ao custo proibitivo dos produtos farmacêuticos. Para um grande número de pessoas pobres da zona rural e urbana nessa região, as plantas medicinais oferecem o único meio de tratamento disponível tanto para as doenças menos graves quanto para as mais sérias (Elisabetsky \& Wannamacher, 1993). Dentre essas, encontra-se a malária que está presente nas regiões tropicais e subtropicais do mundo (FUNASA, 2002).

$\mathrm{Na}$ região Amazônica a maior parte dos casos de malária é causada pelo Plasmodium vivax Grassi \& Feletti 1890, entretanto, o percentual de casos por Plasmodium falciparum Welch 1897 é preocupante, pois é o agente causador da doença na forma mais grave podendo levar a óbitos (Brasil, 2005). Segundo os moradores da Reserva de Desenvolvimento Sustentável do Tupé, a doença que mais atinge os comunitários é a malária (Scudeller et al., 2009) e diante da dificuldade em acessar postos de saúde na cidade, visto que nas comunidades desta reserva não há atendimento médico regular, os moradores ainda utilizam os recursos locais para tratar dessa e de outras enfermidades.

Em relação ao exposto, este estudo se propôs a investigar, resgatar e relatar o conhecimento popular a respeito de plantas medicinais utilizadas no tratamento de malária e males associados pelos moradores da comunidade Julião situada na RDS Tupé, uma unidade de conservação na zona rural do município de Manaus, Amazônia Central.

\section{MATERIAL E MÉTODOS \\ Área de estudo}

A Reserva de Desenvolvimento Sustentável (RDS) do Tupé é a maior unidade de conservação municipal de Manaus com cerca de 12.000 ha (Scudeller et al., 2005), localizada a $25 \mathrm{~km}$ em linha reta do centro da cidade com sede nas coordenadas $03^{\circ} 00^{\prime} 27,47^{\prime \prime}$ 'S e 60'12'14,97'W (SEMMAS, 2007).

Na RDS Tupé existem seis comunidades compostas praticamente por ribeirinhos oriundos do interior do estado sendo a comunidade Julião o foco deste trabalho (Figura 1). Das 72 famílias mencionadas como moradoras efetivas da comunidade, 46 vivem na vila propriamente dita (Souza, 2010). Efetivamente metade destes moradores tem residência fixa na cidade devido à falta de oportunidade de estudo e trabalho para os adolescentes e adultos, e também por causa do período de seca dos rios que dificulta o acesso e os deslocamentos na comunidade.

\section{Oficina e Levantamento Etnobotânico}

As oficinas para levantamento de dados a partir da memória dos moradores sobre as plantas medicinais utilizadas para o tratamento de malária e males associados foram realizadas em outubro de 2008. Entretanto, as visitas (turnês guiadas por quintais e na mata adjacente à comunidade) e as coletas botânicas foram realizadas entre fevereiro de 2007 a julho de 2010. A realização desta técnica consistiu em fundamentar e confirmar os nomes das plantas citadas nas entrevistas (Albuquerque et al., 2010).

O levantamento etnobotânico foi conduzido na forma de oficinas participativas e segregadas por gênero onde os comunitários foram instruídos a lembrar das plantas que utilizavam para fins medicinais e posteriormente segregadas as relatadas para o tratamento de malária e males associados. Porém, houve necessidade de complementar e validar essas informações por meio de entrevistas realizadas de forma individual. Todos os participantes desta pesquisa assinaram o termo de livre consentimento e o estudo foi aprovado pelo

Rev. Bras. PI. Med., Campinas, v.17, n.4, supl. I, p.737-747, 2015. 


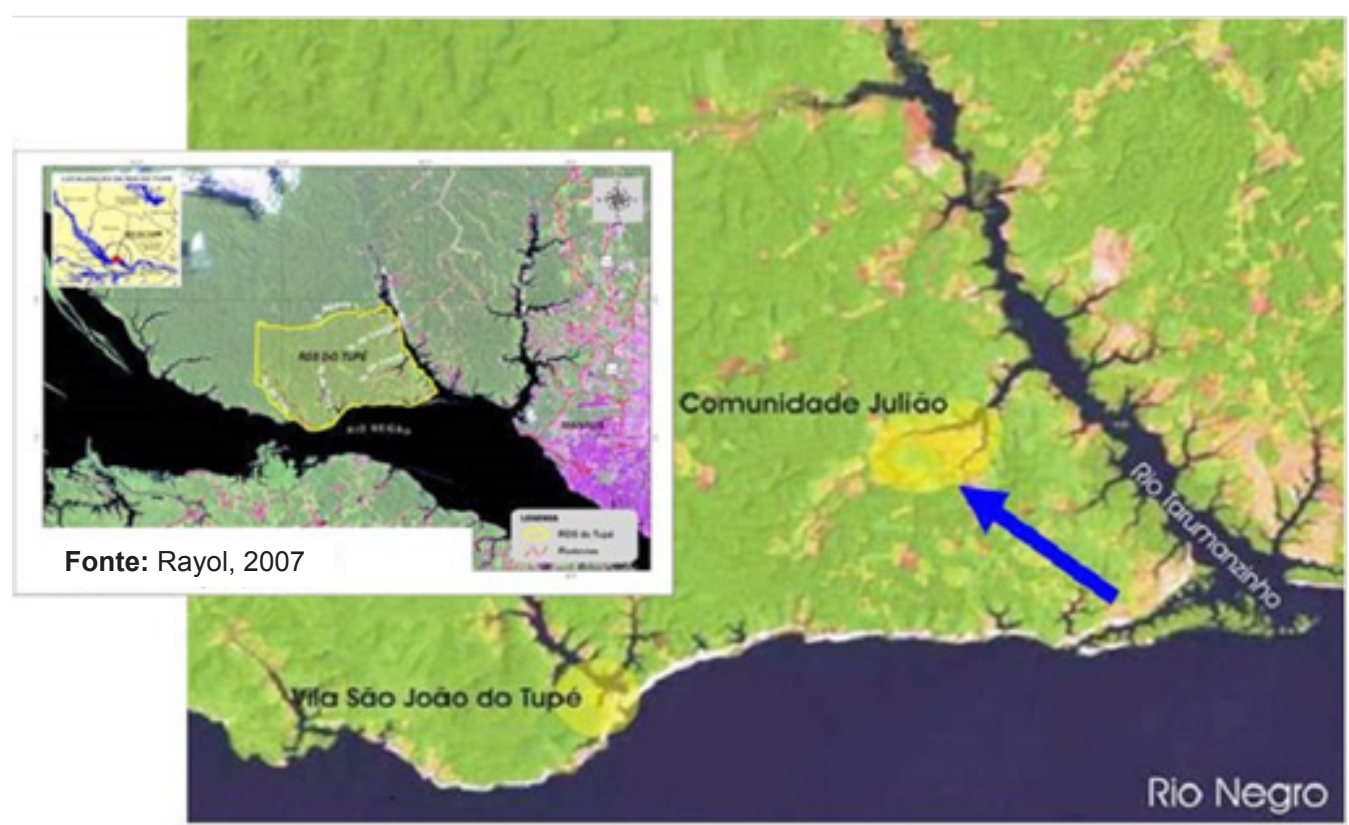

FIGURA 1. Mapa da região do baixo Rio Negro evidenciando os limites da Reserva de Desenvolvimento Sustentável do Tupé a oeste de Manaus. Destaque dado a comunidade Julião com a seta azul.

Comitê de Ética do INPA/CEP (Processo n 158/07) e Conselho de Gestão do Patrimônio Genético CGEN (Processo no 02000.001387/2008-11).

Do total de plantas citadas, aquelas exclusivas para tratar dor de cabeça não foram relacionadas neste estudo, pois este é um sintoma de várias doenças distintas e não poderia ser relacionado apenas à malária e seus males. Em contrapartida, as plantas citadas e utilizadas para "afinar o sangue" ou "limpar o sangue" foram categorizadas como depurativas e computadas neste estudo.

\section{Coleta de material botânico}

As plantas foram classificadas de acordo com o hábito e todo material citado pelos comunitários como medicinal foi coletado e quando fértil, herborizado conforme metodologia convencional (Mori et al., 1989).

A lista de espécies apresentada segue o sistema de classificação APGIII (Angiosperm Phylogeny Group, 2009) e as exsicatas foram depositadas no Herbário INPA. As informações sobre o nome científico e origem foram baseados em: Silva et al. (1977), Lorenzi (1999), Ribeiro et al. (1999), Lorenzi \& Souza (2001), Revilla (2002), Lorenzi \& Matos (2002), IPNI (2013), Kew (2013) e Tropicos (2013).

\section{Análise dos dados}

Para estimar a diversidade do uso dos recursos vegetais na comunidade foi calculado o índice de Shannon-Wiener (Brower et al., 1997) e sua equitabilidade $(\mathrm{J})$.
Para avaliar o Índice de Fidelidade dos usos mencionados para cada espécie foi utilizada a metodologia proposta por Friedman et al. (1986) e modificada por Amorozo \& Gély (1988) que consistiu no cálculo da porcentagem de Concordância quanto aos Usos Principais - CUP - (mais citados) para a espécie, usando-se o número de informantes que citaram o uso principal vezes 100 , dividido pelo número de informantes que citaram a espécie. $\mathrm{E}$ devido às diferenças no número de informantes que citaram os diferentes usos para cada espécie foi necessária a utilização de um Fator de Correção (FC). O Fator de Correção é igual ao número de informantes que citaram usos para a espécie, dividido pelo número de informantes que a citaram como espécie principal, ou seja, com maior número de usos referidos e para calcular a porcentagem de Concordância corrigida quanto aos Usos Principais para cada uma das espécies $\left(\mathrm{CUP}_{\mathrm{C}}\right)$ foi utilizada a multiplicação de CUP e FC (Amorozo \& Gély, 1988).

Foi calculado o Índice de Similiaridade de Jaccard (Magurran, 1988; Brower et al., 1997) entre este estudo e comparado com os demais trabalhos realizados na Amazônia (Milliken,1997; Hidalgo, 2003; Caraballo et al., 2004; Veiga, 2011) e em outras regiões do Brasil (Oliveira et al., 2003; Botsaris, 2007).

\section{RESULTADOS E DISCUSSÃO}

Participaram das oficinas etnobotânicas 13 moradores da comunidade Julião sendo oito homens (62\%) e cinco mulheres (38\%). Nesta etapa, a participação dos comunitários na elaboração da

Rev. Bras. PI. Med., Campinas, v.17, n.4, supl. I, p.737-747, 2015. 
lista livre foi intensa e interativa, pois um comunitário estimulava o outro a lembrar de mais plantas e usos. Dessa forma, foram registradas 62 espécies utilizadas especificamente para o tratamento de malária e males associados, pertencentes a 53 gêneros e 34 famílias botânicas. Destas, apenas dez espécies são nativas da Amazônia e cinco não foram identificadas ao nível específico (Tabela 1). No entanto, o índice de Shannon-Wiener obtido $\left(H^{\prime}=1,62\right.$ decits) representa baixa diversidade de conhecimento em relação ao uso de plantas para o tratamento de malária e males associados. Entretanto a equitabilidade foi de 0,90 indicando que o conhecimento da comunidade em relação as plantas utilizadas é consideravelmente homogêneo. Veiga (2011) ao estudar as plantas utilizadas para tratamento de malária e males associados em uma comunidade vizinha obteve índice de ShannonWiener menor ainda $\left(H^{\prime}=1,18\right.$ decits $)$, porém equitabilidade semelhante $(\mathrm{J}=0,91)$.

As famílias mais ricas em termos de espécies foram: Fabaceae (sete espécies), Asteraceae e Lamiaceae (quatro espécies cada), Solanaceae e Rutaceae (três espécies cada), seguidas por Arecaceae, Annonaceae, Apocynaceae, Euphorbiaceae, Myrtaceae, Poaceae e Zingiberaceae (duas espécies cada) e as demais representadas por uma espécie cada (Tabela 1).

Os homens fizeram o maior número de citações de uso para as plantas relatadas (136) que as mulheres (100). Do total de espécies registradas, 37 foram citadas por ambos os sexos, 19 exclusivamente pelos homens e seis somente pelas mulheres. Estes dados são contrários a alguns estudos onde as mulheres têm demonstrado conhecer uma riqueza maior de plantas em determinados grupos étnicos (Begossi et al., 2002).
Porém, outras pesquisas mostram que o número de espécies conhecidas independe do gênero e da idade (Botrel et al., 2006; Lozada et al., 2006).

Em relação ao hábito das plantas citadas $38,7 \%$ são ervas, $30,6 \%$ árvores, $19,3 \%$ arbustos, $8,1 \%$ lianas e $3,3 \%$ palmeiras (Tabela 1 ). A predominância das herbáceas foi observada em outros estudos realizados na Amazônia por Amorozo \& Gély (1988), Coelho-Ferreira (2000) e Leão et al. (2007). Hidalgo (2003) argumenta que a preferência pelas ervas é explicada pelo seu pequeno porte, facilidade de propagação e cultivo, podendo ser mantidas em canteiros de chão ou suspensas, assim como em diversos tipos de recipientes estando sempre acessíveis. O autor complementa que, no caso de inundações, são as plantas mais fáceis de serem removidas e colocadas a salvo das águas.

Entre os homens o conhecimento de plantas de porte arbóreo é maior $(39,4 \%)$ enquanto que as mulheres demonstraram conhecer mais plantas herbáceas (37,3\%, Figura 2). Sendo assim, os homens, apesar de conhecerem mais espécies arbóreas que as mulheres, relataram um número expressivo de espécies herbáceas, contrário ao apresentado por Silva (2002) que argumenta que homens conhecem mais espécies arbóreas, arbustivas e ruderais que geralmente são encontradas nos caminhos de roças, picadas, matas e roçados ao passo que as mulheres possuem um conhecimento mais específico de ervas e arbustos, espécies cultivadas espontaneamente nas imediações das residências e quintais.

Informações desse tipo são fundamentais para evidenciar como o conhecimento sobre plantas se distribui na comunidade e se este pode ser influenciado por aspectos socioculturais do informante. De acordo com Silva \& Proença

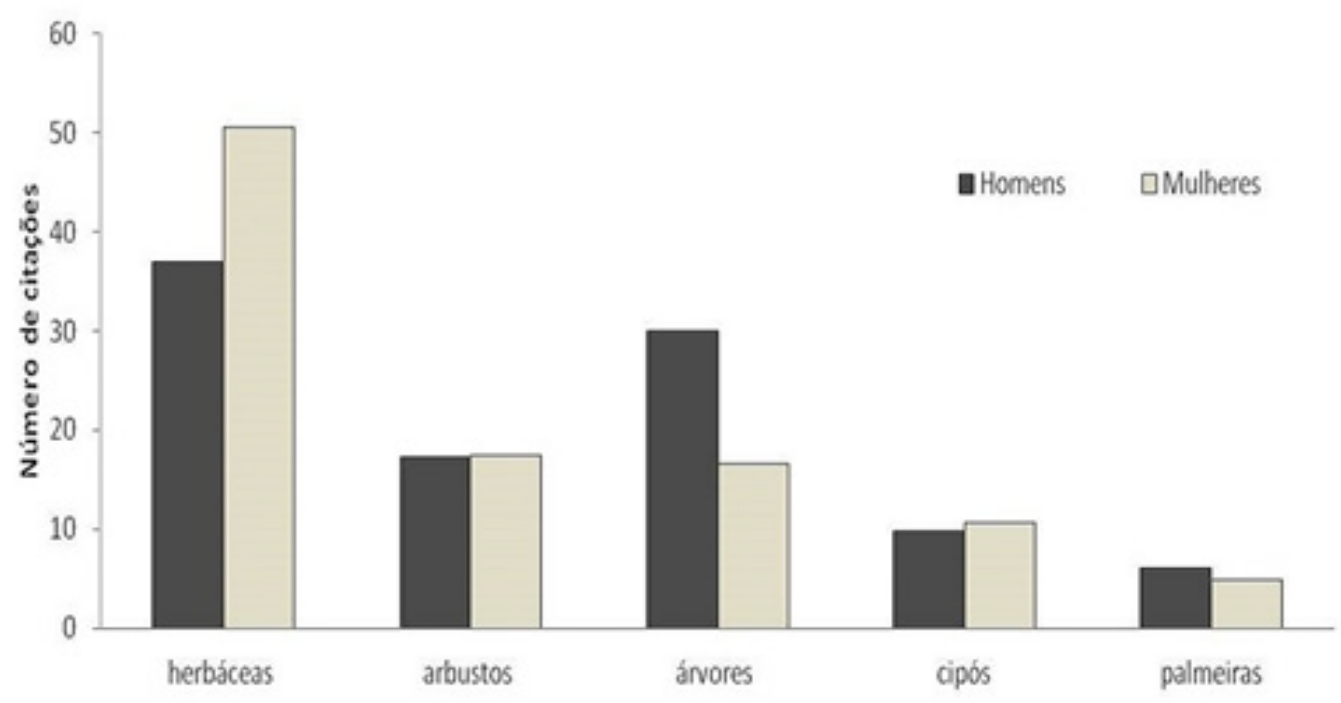

FIGURA 2. Hábito das plantas citadas pelos entrevistados na comundiade Julião (Reserva de Desenvolvimento Sustentável do Tupé)

Rev. Bras. PI. Med., Campinas, v.17, n.4, supl. I, p.737-747, 2015. 
(2008), os processos de decisão do ser humano são extremamente complexos e fatores sociais, culturais, ecológicos e econômicos podem afetar consideravelmente na escolha e no conhecimento de espécies medicinais. Phillips \& Gentry (1993) argumentaram que o conhecimento botânico não constitui uma unidade homogênea entre os sexos, sendo aparentemente determinado pelo papel social que homens e mulheres desempenham ou pelas experiências pessoais adquiridas no cotidiano.

Nessa comunidade, especialmente para malária, o fator determinante é o contato com a doença, pois os maiores conhecedores dos remédios foram os que já tiveram contato com a doença pelo menos uma vez, exceto um comunitário que nunca teve malária.

Apesar da alta porcentagem de espécies citadas para o tratamento de malária e males associados, os comunitários do Julião foram unânimes em dizer que só usam estes preparados após o término da medicação específica prescrita pelo médico e fornecida pelo posto de saúde, ou seja, usam as plantas para amenizar os efeitos colaterais da doença e dos remédios alopáticos geralmente utilizados no tratamento. Estas plantas, por ordem de importância são usadas para tratar o fígado $(19,8 \%)$, anemia $(17,2 \%)$, estômago $(15,5 \%)$, febre $(11,2 \%)$, rins $(6,9 \%)$, gastrite $(5,2 \%)$, depurativas do sangue $(4,3 \%)$ e hepatite $(1,8 \%)$ conforme apresentado na Tabela 1.

As plantas usadas especificamente para tratamento de malária representam $17,2 \%$ enquanto que somente $0,9 \%$ das plantas são utilizadas na prevenção desta doença como é o caso de Ampelozizyphus amazonicus (Tabela 1), e são utilizadas para tratar mais de uma doença. Entretanto, Veiga (2011) na comunidade São João do Tupé (RDS Tupé) para o tratamento de malária e males associados registrou maior valor referente às plantas usadas especificamente para tratamento de malária $(36,6 \%)$.

Provavelmente o método utilizado no levantamento tenha influenciado os resultados obtidos, pois durante a oficina houve interação entre os participantes, aumentando significativamente a riqueza de espécies citadas, porém com diversificados usos e menor detalhamento dos mesmos quando comparado ao levantamento realizado por Veiga (2011) que utilizou entrevistas semiestruturadas aplicadas individualmente e que culminou em uma riqueza muito menor de espécies antimaláricas (apenas 20).

Quanto à parte da planta utilizada no preparado caseiro, as folhas $(51,4 \%)$, caule (cascas, leite e resinas) e raiz (tubérculos e rizoma) $(15,7$ e $14,3 \%$, respectivamente) foram as partes mais citadas, seguidas por frutos $(10 \%)$, sementes
$(5,7 \%)$ e flor $(2,9 \%)$ (Tabela 1$)$. Segundo um dos informantes "é na folha que se encontra a maioria dos princípios ativos da planta" (R.F.B., 74 anos). Estes resultados corroboram os achados de CoelhoFerreira (2000), Amorozo (2002) e Lisboa (2002). Para Hidalgo (2003), o hábito de usar as cascas no preparo de remédios caseiros é amplamente disseminado por toda a Amazônia e em outras áreas do mundo. Segundo o autor, a parte usada é a porção viva da casca e para algumas espécies os informantes referem-se ao uso da entrecasca, a porção mais interna das cascas sendo a preferência por casca recém-coletada; no entanto, quando não se tem acesso fácil à planta, a casca pode ser guardada após secagem para ser usada, por vezes, após vários meses ou anos e as cascas de várias espécies também podem ser compradas em mercados e feiras, nas sedes dos municípios e na capital do estado.

Segundo Vendruscolo \& Mentz (2006), - CUPc pode indicar quais são as espécies mais promissoras para a realização de estudos farmacológicos relativos aos usos indicados pela população. No Julião, as espécies mais promissoras são Geissospermum sericeum e Ampelozizyphus amazonicus (CUPc $=31,25 \%$ cada); pau-pra-tudo (indeterminada) e Croton sacaquinha (CUPc $=25 \%$ cada, Tabela 1$)$. Vale destacar que Ampelozizyphus amazonicus obteve CUPc igual a $50 \%$ na comunidade São João do Tupé (Veiga, 2011), e $53,2 \%$ em levantamento realizado por Hidalgo (2003) e relatada como a espécie com maior concordância de uso. Alguns trabalhos corroboram com o presente estudo como Oliveira et al. (2011) em um levantamento etnobotânico realizado em comunidades quilombolas de Oriximiná, Pará, destacaram a saracuramirá (SAR), Ampelozizyphus amazonicus Ducke com vasto uso popular no tratamento da malária como tônica e depurativa, neste estudo por meio de uma análise etnobotânica quantitativa foi verificado que SAR apresentouse dentre as 10 espécies mais versáteis pela elevada importância relativa $(1,3)$ dentre as cinco espécies com maior importância cultural pelo elevado índice de saliência $(0,311)$ e a espécie com maior concordância de uso principal para malária $(85,7 \%)$. Enquanto que os estudos de Brandão et al. (1992) com G. sericeum também conhecida como "quinarana" ressaltam que a espécie é utilizada tradicionalmente na região amazônica para o tratamento de malária, febre e distúrbios hepáticos.

Quanto às plantas usadas para tratar as doenças associadas à malária foi constatado que - Vernonia condensata é utilizado para tratar o fígado após o tratamento convencional de malária por $68,75 \%$ dos participantes (Tabela 1). Para o tratamento de anemia pós-malária, Persea

Rev. Bras. PI. Med., Campinas, v.17, n.4, supl. I, p.737-747, 2015. 
TABELA 1. Plantas utilizadas pelos moradores da Comunidade Julião - RDS Tupé para o tratamento de malária e males associados de acordo com nome comum, nome científico, família, hábito, origem (provável), usos, parte usada e Concordância Corrigida quanto aos Usos Principais (CUPc). Os usos principais foram destacados em negrito e o número do registro do herbário junto ao nome cientifico.

\begin{tabular}{|c|c|c|c|c|c|c|c|}
\hline Nome comum & Nome científico & Família & Hábito & $\begin{array}{c}\text { Origem } \\
\text { (provável) }\end{array}$ & Usos & Parte usada & CUP $_{c}$ \\
\hline abacate & $\begin{array}{l}\text { Persea americana Mill. } \\
\text { INPA } 234410\end{array}$ & Lauraceae & árvore & $\begin{array}{l}\text { América } \\
\text { Central }\end{array}$ & $\begin{array}{c}\text { anemia, infecção nos rins, fígado, } \\
\text { malária, afinar o sangue }\end{array}$ & folhas & 62,50 \\
\hline açaí & $\begin{array}{c}\text { Euterpe precatoria Mart. } \\
\text { INPA } 234411\end{array}$ & Arecaceae & palmeira & Amazônia & anemia, malária, fígado & raiz & 56,25 \\
\hline algodão-roxo** & Gossypium arboreum L. & Malvaceae & arbusto & América & anemia & folhas & 6,25 \\
\hline amapá $^{\star \star}$ & $\begin{array}{c}\text { Brosimum parinarioides Ducke } \\
\text { INPA } 224465\end{array}$ & Moraceae & árvore & & estômago & leite & 6,25 \\
\hline amor-crescido & Portulaca pilosa L. INPA 234564 & Portulacaceae & erva & América & fígado & folhas & 18,75 \\
\hline anador* & $\begin{array}{c}\text { Plectranthus barbatus Benth. } \\
\text { INPA } 234463\end{array}$ & Lamiaceaae & erva & Índia & febre & folhas & 6,25 \\
\hline babosa & $\begin{array}{l}\text { Aloe vera (L.) Burm. f. } \\
\text { INPA } 234576\end{array}$ & Asphodelaceae & erva & África & estômago, malária & folhas & 6,25 \\
\hline batata-inlesa & Solanum tuberosum $\mathrm{L}$. & Solanaceae & erva & Peru & estômago & tubérculo & 18,75 \\
\hline batatão & indeterminada & & erva & & limpar o sangue & tubérculo & 18,75 \\
\hline beterraba & Beta vulgaris L. & Chenopodiaceae & erva & & anemia, malária & tubérculo & 50 \\
\hline biriba** $^{* *}$ & Rollinia mucosa (Jacq.) Baill. & Annonaceae & árvore & $\begin{array}{l}\text { América do } \\
\text { Sul }\end{array}$ & anemia & fruto & 6,25 \\
\hline $\begin{array}{l}\text { boldo-da-folha- } \\
\text { grande }\end{array}$ & $\begin{array}{c}\text { Vernonia condensata Becker } \\
\text { INPA } 234522\end{array}$ & Asteraceae & arbusto & África & fígado, estômago & folhas & 68,75 \\
\hline $\begin{array}{l}\text { boldo-da-folha- } \\
\text { pequena }\end{array}$ & Plectranthus sp. & Lamiaceae & erva & & fígado & folhas & 50 \\
\hline cana-de-açucar* & Saccharum officinarum L. & Poaceae & erva & $\begin{array}{c}\text { Ásia } \\
\text { meridional }\end{array}$ & anemia & colmo & 6,25 \\
\hline capim-santo & Cymbopogon citratus (DC.) Stapf. & Poaceae & erva & Europa & estômago, febre & folhas & 6,25 \\
\hline carapanaúba & Aspidosperma sp. & Apocynaceae & árvore & Amazônia & fígado, estômago, anemia, malária & casca & 37,50 \\
\hline carmelitana* $^{*}$ & Indeterminada & & arbusto & & estômago & folhas & 6,25 \\
\hline cibalena** & Indeterminada & & erva & & febre & folhas & 12,50 \\
\hline cipó-tuíra & $\begin{array}{c}\text { Bonamia ferruginea (Choisy) } \\
\text { Hallier f. INPA } 229655\end{array}$ & Convolvulaceae & liana & Amazônia & $\begin{array}{l}\text { rins, malária,anemia, } \\
\text { fígado, afinar o sangue }\end{array}$ & folha, ramos & 31,25 \\
\hline coco & Cocos nucifera L. & Arecaceae & palmeira & Ásia & hepatite, anemia & fruto & 6,25 \\
\hline copaíba** $^{*}$ & Copaifera sp. & Fabaceae & árvore & $\begin{array}{l}\text { América do } \\
\text { Sul }\end{array}$ & febre & óleo & 6,25 \\
\hline corama & $\begin{array}{c}\text { Bryophyllum calycinum Salisb. } \\
\text { INPA } 213345\end{array}$ & Crassulaceae & erva & & estômago & folhas & 12,50 \\
\hline couve & $\begin{array}{l}\text { Brassica oleracea L. var. } \\
\text { acephala }\end{array}$ & Brassicaceae & erva & Mediterrâneo & anemia, estômago & folhas & 18,75 \\
\hline crajirú & $\begin{array}{c}\text { Arrabidaea chica (H. \& B.) Verlot } \\
\text { INPA } 245677\end{array}$ & Bignoniaceae & liana & Amazônia & anemia & folhas & 31,25 \\
\hline cubiu $^{* *}$ & Solanum sessiliflorum Dunal & Solanaceae & arbusto & Amazônia & anemia & fruto & 6,25 \\
\hline cumaru $^{* *}$ & Dipteryx odorata (Aubl.) Willd. & Fabaceae & árvore & $\begin{array}{l}\text { América do } \\
\text { Sul }\end{array}$ & estômago & semente & 6,25 \\
\hline $\begin{array}{c}\text { embaúba } \\
\text { branca }\end{array}$ & Cecropia sp. & Cecropiaceae & árvore & & malária, fígado, rins & $\begin{array}{r}\text { semente, } \\
\text { folhas }\end{array}$ & 12,50 \\
\hline $\begin{array}{c}\text { erva-de- } \\
\text { passarinho** }\end{array}$ & Phthirusa pyrifolia (H.B.K.) Eich. & Loranthaceae & erva & & estômago & folha, ramos & 6,25 \\
\hline escada-de-jabuti & Bauhinia guianensis Aubl. & Fabaceae & liana & & rins, malária, fígado & casca & 18,75 \\
\hline eucalipto & Eucalyptus sp. & Myrtaceae & árvore & & febre & folhas & 31,25 \\
\hline feijão** & Phaseolus vulgaris $\mathrm{L}$. & Fabaceae & erva & $\begin{array}{l}\text { América do } \\
\text { Sul }\end{array}$ & anemia & semente & 6,25 \\
\hline graviola & Annona muricata L. & Annonaceae & árvore & $\begin{array}{l}\text { América } \\
\text { Tropical }\end{array}$ & anemia & folhas & 12,50 \\
\hline hortelãzinho** & Mentha piperita L. & Lamiaceae & erva & Europa & estômago & folhas & 6,25 \\
\hline jambo** & Eugenia malaccensis L. & Myrtaceae & árvore & Ásia & anemia & folhas & 12,50 \\
\hline jambú & $\begin{array}{c}\text { Spilanthes acmella (L.) Murray } \\
\text { INPA } 234420\end{array}$ & Asteraceae & erva & $\begin{array}{l}\text { América do } \\
\text { Sul }\end{array}$ & fígado & folha, flor & 12,50 \\
\hline
\end{tabular}


TABELA 1. Plantas utilizadas pelos moradores da Comunidade Julião - RDS Tupé para o tratamento de malária e males associados de acordo com nome comum, nome científico, família, hábito, origem (provável), usos, parte usada e Concordância Corrigida quanto aos Usos Principais (CUPc). Os usos principais foram destacados em negrito e o número do registro do herbário junto ao nome cientifico.

\begin{tabular}{|c|c|c|c|c|c|c|c|}
\hline jenipapo & Genipa americana L. & Rubiaceae & árvore & $\begin{array}{l}\text { América do } \\
\text { Sul }\end{array}$ & anemia & fruto & 56,25 \\
\hline jurubeba* & Solanum paniculatum L. & Solanaceae & arbusto & $\begin{array}{l}\text { América do } \\
\text { Sul }\end{array}$ & fígado & raiz & 6,25 \\
\hline laranja & $\begin{array}{l}\text { Citrus sinensis (L.) Osbeck } \\
\text { INPA } 234589\end{array}$ & Rutaceae & árore & Ásia & estômago, fígado & $\begin{array}{l}\text { casca do } \\
\text { fruto }\end{array}$ & 25 \\
\hline laranja-da-terra & Citrus cf. aurantium L. & Rutaceae & árvore & Ásia & depurativo, anemia & fruto & 18,75 \\
\hline $\operatorname{limão**}$ & Citrus limon (L.) Burm. F. & Rutaceae & arbusto & Ásia & febre & folhas & 6,25 \\
\hline macela & $\begin{array}{l}\text { Achyrocline satureioides D.C. } \\
\text { INPA } 224387\end{array}$ & Asteraceae & erva & Amazônia & estômago, febre, malária & folhas & 18,75 \\
\hline mamão & Carica papaya L. & Caricaceae & arbusto & $\begin{array}{l}\text { América } \\
\text { Central }\end{array}$ & fígado, malária & folha, flor & 6,25 \\
\hline mangarataia** $^{* *}$ & $\begin{array}{c}\text { Zingiber officinale Roscoe } \\
\text { INPA } 234425\end{array}$ & Zingiberaceae & erva & Ásia & estômago & rizoma & 6,25 \\
\hline mangirioba** $^{\star *}$ & Cassia ocidentalis L. & Fabaceae & árvore & $\begin{array}{l}\text { América } \\
\text { Tropical }\end{array}$ & $\begin{array}{l}\text { malária, anemia, limpar o sangue, } \\
\text { febre, hepatite }\end{array}$ & raiz & 12,50 \\
\hline $\begin{array}{l}\text { melão-de-são- } \\
\text { caetano** }\end{array}$ & Momordica charantia L. & Cucurbitaceae & liana & Ásia & malária, fígado & $\begin{array}{c}\text { fruto, } \\
\text { semente }\end{array}$ & 12,50 \\
\hline melhoral & Artemisia vulgaris $\mathrm{L}$. & Asteraceae & erva & Europa/Ásia & febre, estômago & folhas & 25 \\
\hline murici** & $\begin{array}{l}\text { Byrsonima chrysophylla Kunth } \\
\text { INPA } 234409\end{array}$ & Malpighiaceae & árvore & $\begin{array}{l}\text { América do } \\
\text { Sul }\end{array}$ & estômago & casca & 6 \\
\hline óleo elétrico & Piper cf. callosum Ruiz \& Pav. & Piperaceae & arbusto & & estômago & folhas & 18,75 \\
\hline oriza $^{* *}$ & Pogostemon patchouly Pellet & Lamiaceae & erva & Ásia & estômago & folhas & 6,25 \\
\hline pata-de-vaca** $^{* *}$ & Bauhinia forticata Link & Fabaceae & árvore & $\begin{array}{l}\text { América do } \\
\text { Sul }\end{array}$ & malária & folhas & 6,25 \\
\hline pau-prá-tudo & indeterminada & & árvore & & malária,fígado,estômago & casca, raiz & 25 \\
\hline pobre-velho & $\begin{array}{l}\text { Costus spicatus (Jacq.) Sw. } \\
\text { INPA } 225567\end{array}$ & Costaceae & erva & $\begin{array}{l}\text { América do } \\
\text { Sul }\end{array}$ & rins, fígado & folha & 37,50 \\
\hline quebra-pedra & $\begin{array}{l}\text { Phyllanthus niruri L. } \\
\text { INPA } 225698\end{array}$ & Phyllanthaceae & erva & $\begin{array}{l}\text { América } \\
\text { Tropical }\end{array}$ & rins, fígado & raiz, folhas & 56,25 \\
\hline quina-quina & $\begin{array}{l}\text { Geissospermum sericeum Benth. } \\
\text { \& Hook. f. ex Miers INPA } 234580\end{array}$ & Apocynaceae & árvore & $\begin{array}{l}\text { América do } \\
\text { Sul }\end{array}$ & malária, fígado, febre & casca & 31,25 \\
\hline sacaca & $\begin{array}{l}\text { Croton cajucara Benth. } \\
\text { INPA } 234590 \\
\end{array}$ & Euphorbiaceae & arbusto & Amazônia & $\begin{array}{c}\text { fígado, malária, febre, infecção nos } \\
\text { rins }\end{array}$ & folhas & 31,25 \\
\hline sacaquinha & $\begin{array}{l}\text { Croton sacaquinha Croizat } \\
\text { INPA } 229653\end{array}$ & Euphorbiaceae & arbusto & Amazônia & $\begin{array}{l}\text { malária, febre, fígado, infecção nos } \\
\text { rins, estômago }\end{array}$ & folhas & 25 \\
\hline salva-de-marajó & Indeterminada & & arbusto & & estômago, fígado & folhas & 25 \\
\hline saracura-mirá & $\begin{array}{c}\text { Ampelozizyphus amazonicus } \\
\text { Ducke INPA } 234596\end{array}$ & Rhamnaceae & liana & Amazônia & $\begin{array}{l}\text { preventivo malária, } \\
\text { malária, fígado }\end{array}$ & casca & 31,25 \\
\hline sena $^{* *}$ & Senna alexandrina Mill. & Fabaceae & arbusto & Africa & fígado & folhas & 6,25 \\
\hline terramicina* & $\begin{array}{c}\text { Alternanthera brasiliana (L.) } \\
\text { Kuntze }\end{array}$ & Amaranthaceae & erva & $\begin{array}{l}\text { América do } \\
\text { Sul }\end{array}$ & Febre & folhas & 6,25 \\
\hline vindicá & $\begin{array}{c}\text { Alpinia speciosa (Blume) D. Dietr. } \\
\text { INPA } 234428\end{array}$ & Zingiberaceae & erva & Ásia & Febre & folhas & 6,25 \\
\hline xixuá & $\begin{array}{c}\text { Maytenus guianensis Klotzch. } \\
\text { INPA } 234517\end{array}$ & Celastraceae & árvore & Amazônia & malária, anemia & casca & 12,50 \\
\hline
\end{tabular}

americana foi a espécie que apresentou maior valor de concordância $62,50 \%$, seguida por Euterpe precatoria, Phyllanthus niruri e Genipa americana todos com CUPc igual a $56,25 \%$ (Tabela 1). Na comunidade São João do Tupé, Euterpe precatoria é indicada para o tratamento de anemia pós-malária por $100 \%$ dos entrevistados enquanto Persea americana obteve CUPc igual a $25 \%$ para a mesma finalidade (Veiga, 2011). Existem estudos relatando a eficiência do extrato aquoso da folha de Persea americana para diabetes (Marrero-Faz et al., 2014), contra Plasmodium falciparum (Falodum et al., 2014), antimicrobactéria e antiprotozoários (JimenezArellana, et al., 2013); atividade anticonceptiva e anti-inflamatória de Vernonia condensata (Silva et al., 2011), ação toxicológica da folha (Monteiro et al.,

Rev. Bras. PI. Med., Campinas, v.17, n.4, supl. I, p.737-747, 2015. 
TABELA 2. Comparação entre seis levantamentos etnobotânicos voltados aos estudos de plantas utilizadas no tratamento de malária e males associados realizados na América Latina.

\begin{tabular}{lccccccc}
\hline & $\begin{array}{l}\text { Este } \\
\text { trabalho }\end{array}$ & $\begin{array}{c}\text { Veiga } \\
(2011)\end{array}$ & $\begin{array}{c}\text { Botsaris } \\
(2007)\end{array}$ & $\begin{array}{c}\text { Caraballo } \\
\text { et al. (2004) }\end{array}$ & $\begin{array}{c}\text { Hidalgo } \\
(2003)\end{array}$ & $\begin{array}{c}\text { Oliveira et al. } \\
(2003)\end{array}$ & $\begin{array}{c}\text { Milliken } \\
(1997)\end{array}$ \\
\hline Local & RDS & RDS & & & & 7 municípios & \\
Tupé & Tupé & Revisão & Venezuela & Amazonas & Revisão & Roraima \\
\hline Total & 62 & 114 & 40 & 18 & 126 & 197 & 99 \\
Antimaláricas & 62 & 20 & 40 & 18 & 82 & 197 & 65 \\
Famílias & 34 & 15 & 21 & 14 & 53 & 63 & 41 \\
Espécies/comum & 15 & 15 & 3 & 4 & 34 & 16 & 11 \\
Famílias/comum & 11 & 11 & 3 & 4 & 25 & 12 & 9 \\
Jaccard & & 15,46 & 2,85 & 4,76 & 9,10 & 5,47 & 7,97 \\
\hline
\end{tabular}

Espécies/comum = espécies em comum entre os levantamentos

Famílias/comum = famílias em comum entre os levantamentos

2011); atividade antioxidante de Euterpe precatoria (Kang et al., 2011), antioxidante e citotóxica (Galotta et al., 2008) e caracterização química (Galotta \& Boaventura, 2005); e um estudo das propriedades farmacológicas (Bagalkotkar et al., 2006). Conforme Friedman et al. (1986) quando uma espécie apresenta maior consenso, supõe-se que esta foi submetida a uma seleção através do tempo, por ensaio e erro e, portanto, a probabilidade é que seja efetivamente melhor.

Dentre as 62 espécies citadas para o tratamento de malária e males associados, 16 espécies (Tabela 1) não foram encontradas em nenhuma das listas utilizadas para comparação. Dentre as plantas informadas pelos comunitários apenas Bauhinia forticata é citada para tratar especificamente a malária. As outras 15 espécies investigadas: Gossypium arboreum, Rollinia mucosa, Saccharum officinarum, carmelitana (indeterminada), cibalena (indeterminada), Solanum sessiliflorum, Phthirusa pyrifolia, Phaseolus vulgaris, Eugenia malaccensis, Zingiber officinale, Pogostemon patchouly, salva-de-marajó (indeterminada), Senna alexandrina, Alternanthera brasiliana e Alpinia speciosa são citadas para tratar os outros males associados à doença. Dessas, cinco são asiáticas, três da América do Sul, uma africana e uma do continente americano e apenas uma da Amazônia, além de quatro sem determinação de origem, ou seja, mais uma expressão da erosão do conhecimento local em detrimento do global.

É comum em levantamentos etnobotânicos da Amazônia e até mesmo de outras partes do Brasil apresentar uma lista de plantas medicinais com pelo menos $50 \%$ de espécies exóticas (Hidalgo, 2003; Souza, 2010), plantas trazidas provavelmente com os colonizadores e disseminada na cultura local. Também vale destacar que a pata-de-vaca (Bauhinia forficata) e o feijão (Phaseolus vulgaris L.) não foram encontrados na comunidade in vivo, mas são frequentemente vendidos nos mercados da cidade e conhecidos pelos comunitários.

A similaridade encontrada entre o levantamento realizado na comunidade Julião com os demais estudos realizados sobre etnobotânica de antimaláricos é baixa (Tabela 2). O estudo que apresentou maior número de espécies em comum (34 spp. e 25 famílias botânicas) foi o de Hidalgo (2003) realizado em sete municípios no Estado do Amazonas. Dentre as espécies comuns pode-se citar Ampelozizyphus amazonicus, Croton cajucara, Persea americana, Euterpe precatoria e Aspidosperma sp. Já quando comparado a outro estudo realizado na mesma RDS (Veiga, 2011), a similaridade é um pouco menor $(15,46 \%)$, apenas 15 espécies e 11 famílias em comum. Mesmo na revisão de Oliveira et al. (2003) e Botsaris (2007) para o Brasil, o número de espécies em comum foi baixo e a similaridade menor ainda (Tabela 2).

Curioso notar que a similaridade com o estudo realizado em Roraima (Milliken 1997) foi maior que os dois estudos de revisão anteriormente citados seguido por Caraballo et al. (2004) (na Venezuela), mostrando que provavelmente a proximidade geográfica e pertencer ao mesmo bioma pode contribuir para o aumento da similaridade do conhecimento etnobotânico, independente da cultura ou etnia estudada. Destaque também para a elevada riqueza de espécies antimaláricas referenciadas no presente estudo, pois somente é menor que os estudos de Oliveira et al. (2003) (revisão do Brasil), Hidalgo (2003) (para 7 municípios do Amazonas) e Milliken (1997) (Roraima com 7 grupos indígenas da savana e floresta e com a população Luso-brasileira) (Tabela 2).

Diante dos resultados obtidos, percebe- 
se que os moradores da comunidade do Julião possuem conhecimento satisfatório sobre plantas medicinais, principalmente as utilizadas para tratamento de malária e seus males. Entretanto, devido a proximidade com a capital do estado e a possibilidade de um tratamento mais rápido e eficaz no tratamento de malária, eles apenas fazem uso destas plantas para tratar as consequências da doença.

Dessa forma, o presente estudo destaca a necessidade de mais pesquisas sobre o assunto, principalmente porque das 62 espécies relatadas como antimaláricas, apenas 10 são nativas da Amazônia, ou seja, poucas espécies conhecidas e utilizadas diante da imensa biodiversidade amazônica e 16 são novos relatos para este fim, pelo menos em comparação com estudos realizados na América Latina.

\section{AGRADECIMENTOS}

Aos moradores da Comunidade Julião (RDS Tupé), Projeto BIOTUPÉ, Programa de PósGraduação em Botânica (INPA), Projeto Saberes Locais (Processo 551988/2007), CT - Amazônia 27 (Processo 553373/2005-6) e ao CNPq pela concessão da bolsa de Doutorado a primeira autora.

\section{REFERÊNCIAS}

ALBUQUERQUE, U.P. et al. Métodos e técnicas para coleta de dados etnobotânicos. In: ALBUQUERQUE, U. P.; LUCENA, R. F. P.; CUNHA, L. V. F. C. (Orgs.). Métodos e técnicas na pesquisa etnobotânica. 1. ed. Recife: NUPPEA. 2010. p.39-64

AMOROZO, M.C.M.; GÉLY, A.L. Uso de plantas medicinais por caboclos do baixo Amazonas, Barcarena, PA, Brasil. Boletim do Museu Paraense "Emílio Goeldi". Nova Série Botânica v.4, n.1, p.47-131, 1988.

AMOROZO, M.C.M. Uso e diversidade de plantas medicinais em Santo Antônio do Leverger, MT, Brasil. Acta Botanica Brasilica, v.16, n.2, p.189-203, 2002.

APG III. An update of the Angiosperm Phylogeny Group classification for the orders and families of flowering plants: APG III. Botanical Journal of the Linnean Society, v.161, p.105-121, 2009.

BAGALKOTKAR, G. et al. Phytochemicals from Phyllanthus niruri Linn. and their pharmacological properties: a review. Journal of Pharmacy and Pharmacology, v. 58, n. 12, p. 1559-1570, 2006.

BALICK, M.; COX, P. Plants, peoples and culture: the science of ethnobotany. $2^{\mathrm{a}}$ ed. New York: Scientific American Library. 1997. 228p.

BALICK, M.J. et al. Medicinal plants used by Latino healers for women's health Conditions. Economic Botany, v.54, n.3, p.344-357, 2000.

BEGOSSI, A. et al. 2002. Ecologia humana, etnoecologia e conservação. In: AMOROZO, M. C. M.; MING, L. C.;
SILVA, S. M. P. (Org.). Métodos de coleta e análise de dados em etnobiologia, etnoecologia e disciplinas correlatas. Rio Claro: UNESP/CNPq, 2002. p.93-128.

BOTREL, R.T. et al. Uso da vegetação nativa pela população local no município de Ingaí, MG, Brasil. Acta Botanica Brasilica, v.20, n. 1, p.143-156, 2006.

BOTSARIS, A.S. Plants used traditionally to treat malaria in Brazil: the archives of Flora Medicinal. Journal of Ethnobiology and Ethnomedicine, v. 3, n. 18, p. 1-8, 2007.

BRANDÃO, M.G.L. et al. Survey of medicinal plants used as antimalarials in the Amazon. Journal of Ethnopharmacology, v. 36, p. 175-82, 1992.

BRASIL. MINISTÉRIO DASAÚDE/Secretaria de Vigilância em Saúde. Malária: Guia de Vigilância Epidemiológica. 6 ed. Brasília: SVS, 2005. 531p.

BRASIL. Ministério da Saúde. RENISUS - Relação Nacional de Plantas Medicinais de Interesse ao SUS. Espécies vegetais. DAF/SCTIE/MS - RENISUS - 2009. Disponível em: <http://portal.saude.gov.br/portal/ arquivos/pdf/RENISUS.pdf>. Acesso: em 20 jul.2012

BROWER, J.H.; ZAR, C.N.; VON ENDE, C.N. Field and laboratory methods for general ecology. $1^{\mathrm{a}} \mathrm{ed}$. United States of America: The Mcgraw-Hill Companies, 1997. 273p.

CARABALLO, A. et al. Avaliação preliminar de plantas medicinais usadas como antimaláricas no sudeste amazônico Venezuelano. Revista da Sociedade Brasileira de Medicina Tropical, v.37, n.2, p.186-188, 2004.

COELHO-FERREIRA, M. R. Identificação e valorização das plantas medicinais de uma comunidade pesqueira do litoral paraense (Amazônia brasileira). 2000. 269 p. Tese (Doutorado em Botânica) Universidade Federal do Pará, Museu Paraense Emílio Goeldi/EMBRAPA, Belém.

CUNNINGHAM, A.B. Ethics, ethnobiological research, and biodiversity. $1^{\text {a }}$ ed. Gland, Switzerland: WWF International publications. B. Lindsay (ed.).1993. 44p.

ELISABETSKY, E.; WANNAMACHER, L. The Status of Ethnopharmacology in Brazil. Journal of Ethnopharmacology, v.38, p.137-143, 1993.

FALODUN, A. et al. Evaluation of three medicinal plant extracts against Plasmodium Falciparum and selected microganisms. African Journal of Traditional Complementary and Alternative Medicines, v. 11, $\mathrm{n}$. 4, p. 142-146, 2014.

FRIEDMAN, J. et al. A preliminary classification of the healing potential of medicinal plants, based on a rational analysis of an ethnopharmacological field survey among Bedouins in the Negev Desert, Israel. Journal of ethnopharmacology, v.16, p.275-287, 1986.

FUNASA - Fundação Nacional de Saúde. Guia de Vigilância Epidemiológica. 5 ed. Brasília: Ministério da Saúde, 2002. 559p.

GALOTTA, A.L.Q.; BOAVENTURA, M.A.D. Chemical constituents from roots and leaf stalks of açai (Euterpe precatoria Mart., Arecaceae). Química Nova, v. 28, n.4, p. 610-613, 2005.

GALOTTA, A.L.Q.A. et al. Antioxidant and cytotoxic activities of 'açai' (Euterpe precatoria Mart.). Química Nova, v. 31, n.6, p. 1427-1430, 2008.

GOTTLIEB, O. R.; KAPLAN, M.A.C.; BORIN, M.R.B.

Rev. Bras. PI. Med., Campinas, v.17, n.4, supl. I, p.737-747, 2015. 
Biodiversidade: um enfoque químico-biológico. $1^{\text {a }} \mathrm{ed}$. Rio de Janeiro: Editora da UFRJ. 1996. 267p.

HIDALGO, A.F. Plantas de uso popular para o tratamento da malária e males associados das áreas de influência do Rio Solimões e região de ManausAM. 2003. 202p. Tese (Doutorado em Agronomia) - Universidade Estadual Paulista Júlio de Mesquita Filho, Botucatu.

IPNI. The International Plant Name Index. Disponível em: <http://www.ipni.org>. Acesso em: 12 mar. 2013.

JIMENEZ-ARELLANES, A. et al. Antiprotozoal and antimycobacterial activities of Persea americana seeds. BMC Complementary and Alternative Medicine, v. 13, n. 109, 2013.

KANG, J. et al. Antioxidant and anti-inflammatory activities of açai (Euterpe precatoria) fruit pulp. Faseb Journal, v. 25, n.1, p. 35- 46, 2011.

KEW. The Royal Botanic Gardens. Disponível em: <http://www.kew.org>. Acesso em: 20 abr. 2013.

LEÃO, R.B.M. et al. Levantamento de plantas de uso terapêutico no município de Santa Bárbara do Pará, Estado do Pará, Brasil. Revista Brasileira de Farmacognosia, v.88, n.1, p.21-25, 2007.

LISBOA, P.L.B. Natureza, homem e manejo de recursos naturais na região de Caxiuanã, Melgaço, Pará. $1^{a}$ ed. Belém: Museu Paraense Emílio Goeldi, 2002. 237p. il.

LORENZI, H. Árvores brasileiras: manual de identificação e cultivo de plantas arbóreas nativas do Brasil. $2^{\mathrm{a}}$ ed. Nova Odessa: Plantarum, 1999. 352 p.

LORENZI, H.; SOUZA, H.M. Plantas ornamentais no Brasil. $1^{a}$ ed. Nova Odessa: Plantarum, 2001. 1088 p.

LORENZI, H.; MATOS, F.J.A. Plantas medicinais no Brasil: nativas e exóticas cultivadas. $1^{\mathrm{a}}$ ed. Nova Odessa: Plantarum, 2002. 512 p.

LOZADA, M.; LADIO, A.; WEIGANDT, M. Cultural transmission of ethnobotanical knowledge in a rural community of northwestern Patagonia, Argentina. Economic Botany, v.60, p.374-385, 2006.

MAGURRAN, A.E. Ecological Diversity and its Measurements. $1^{\text {a }}$ ed. Cambridge: University Press, 1988. 179 p.

MARRERO-FAZ, E. et al. Inhibitory effect of Persea americana Mill. leaf aqueous extract and its fractions on PTP1B as therapeutic target for type 2 diabetes. Boletin Latinoamericano y del Caribe de Plantas Medicinales y Aromaticas, v. 13, n. 2, p. 144-151, 2014.

MILLIKEN, W. Traditional anti-malarial medicine in Roraima, Brazil. Economic Botany, v.3, n.51, p.212237, 1997.

MONTEIRO, M.H.D.; et al. Toxicological evaluation of a tea from leaves of Vernonia condensate. Journal of Ethnopharmacology, v.74, n.2, p. 149-157, 2001.

MORI, S. A.; RABELO, B. V.; TSOU, C.; DALY, D. Composition and structure of Camaipi forest, Brazil. Boletim do Museu Paraense Emílio Goeldi, Série Botânica, v.5, n.1, p.3-18, 1989.

OLIVEIRA, F.Q. et al. Potencial das plantas medicinais como fonte de novos antimaláricos: espécies indicadas na bibliografia etnomédica brasileira. Revista Brasileira de Plantas Medicinais, v.5, n.2, p.23-31, 2003.

OLIVEIRA, D. R. et al. Estudo etnofarmacognóstico da saracuramirá (Ampelozizyphus amazonicus
Ducke), uma planta medicinal usada por comunidades quilombolas do Município de Oriximiná-PA, Brasil. Acta amazonica, v. 41, n. 3, p. 383 - 392, 2011.

PHILLIPS, O.; GENTRY, A.H. The useful plants of Tambopata, Peru. I. Statistical hypotheses with a new quantitative technique. Economic Botany, v.47, n.1, p.33-43, 1993.

PLOTKIN, M. J. The importance of ethnobotany for tropical forest conservation. In: SCHULTES, R. E.; VON REIS, S. (Eds.). Ethnobotany. Portland: Dioscorides Press,1995. p.147- 156.

REVILLA, J. Plantas úteis da bacia amazônica. Manaus: SEBRAE/INPA. v. 2. 2002. 445p.

RIBEIRO, J.E.L.S. et al. Flora da Reserva Ducke: Guia de Identificação das Plantas Vasculares de uma Floresta de Terra Firme na Amazônia Central. $1^{\mathrm{a}} \mathrm{ed}$. Manaus: INPA. 1999. 816p.

SEMMAS. Secretaria Municipal de Meio Ambiente e Sustentabilidade. Plano de Manejo da Reserva de Desenvolvimento Sustentável do Tupé, v. 1, n.1, p. 1-27, 2007. Disponível em: <www2.manaus.am.gov. $\mathrm{br} /$ secretarias/semma/planoManejoTupé>Acesso em: 21 maio 2009.

SCUDELLER, V.V.; APRILE, F.M.; MELO, S.; SANTOSSILVA, E.N. Reserva de Desenvolvimento Sustentável do Tupé: Características gerais. In: SANTOS-SILVA, E.N.; APRILE, F.M.; SCUDELLER, V.V.; MELO, S. (Orgs.). BIOTUPÉ: meio físico, diversidade biológica e sociocultural do baixo Rio Negro, Amazônia Central. Manaus: Ed. INPA, 2005. p. xi

SCUDELLER, V.V.; VEIGA, J.B.; ARAÚJO-JORGE, L.H. Etnoconhecimento de plantas de uso medicinal nas comunidades São João do Tupé e Central (Reserva de Desenvolvimento Sustentável do Tupé). In: SANTOS-SILVA, E.N.; SCUDELLER, V.V. (Orgs.). BIOTUPÉ: meio Físico, Diversidade Biológica e Sociocultural do Baixo Rio Negro, Amazônia Central, vol. 2. Manaus: UEA Edições, 2009. p. 185-199.

SILVA, M.F. et al. Nomes vulgares de plantas amazônicas. $1^{a}$ ed. Belém: INPA, 1977. 222 p. il.

SILVA, R.B.L. A etnobotânica de plantas medicinais da comunidade quilombola de Curiaú, Macapá, Amapá, Brasil. 2002. 172 p. Dissertação (Mestrado em Agronomia), Faculdade de Ciências Agrárias do Pará, Belém.

SILVA, C.S.P.; PROENÇA, C.E.B. Uso e disponibilidade de recursos medicinais no município de Ouro Verde de Goiás, GO, Brasil. Acta Botanica Brasilica, v. 22, n. 2, p. 481-492, 2008.

SILVA, J.B. et al. New approaches to clarify antinociceptive and anti-inflammatory effects of the ethanol extract from Vernonia condensata leaves. International Journal of Molecular Sciences, v. 12, n.12, p. 8993-9008, 2011.

SOUZA, C.C.V. Etnobotânica de quintais em três comunidades ribeirinhas na Amazônia Central, Manaus-AM. 2010. 91p. Dissertação (Mestrado em Botânica) - Instituto Nacional de Pesquisas da Amazônia/Universidade Federal do Amazonas, Manaus.

TROPICOS. Tropicos.org. Missouri Botanical Garden. Disponível em: <http://www. tropicos.org>. Acesso em: 20 abr. 2013.

VEIGA, J.B. Etnobotânica e etnomedicina na Reserva 
de Desenvolvimento Sustentável do Tupé, baixo rio Negro: plantas antimaláricas, conhecimentos e percepções associadas ao uso e à doença. 2011. 154p. Tese (Doutorado em Botânica) - Instituto Nacional de Pesquisas da Amazônia/Universidade Federal do Amazonas, Manaus.
VENDRUSCOLO, G.S.; MENTZ, L.A. Estudo da concordância das citações de uso e importância das espécies e famílias utilizadas como medicinais pela comunidade do bairro Ponta Grossa, Porto Alegre, RS, Brasil. Acta Botanica Brasilica, v.20, n.2, p.367-382, 2006.

Rev. Bras. PI. Med., Campinas, v.17, n.4, supl. I, p.737-747, 2015. 